\title{
Towards quantitative demineralization imaging for the assessment of carious lesions based on PS-OCT
}

\author{
Jonas Golde ${ }^{1, *}$, Florian Tetschke ${ }^{1,2}$, Robin Vosahlo ${ }^{2}$, Lars Kirsten ${ }^{1}$, Julia Walther ${ }^{1,3}$, Christian Hannig ${ }^{2}$, and Edmund \\ $\mathrm{Koch}^{1}$ \\ ${ }^{1}$ Clinical Sensoring and Monitoring, Carl Gustav Carus Faculty of Medicine, TU Dresden, Fetscherstrasse 74, 01307 Dresden, Germany \\ ${ }^{2}$ Policlinic of Operative and Pediatric Dentistry, Carl Gustav Carus Faculty of Medicine, TU Dresden, Fetscherstrasse 74, 01307 \\ Dresden, Germany \\ ${ }^{3}$ Department of Medical Physics and Biomedical Engineering, Carl Gustav Carus Faculty of Medicine, TU Dresden, Fetscherstrasse \\ 74, 01307 Dresden, Germany
}

\begin{abstract}
Assessing the stage and progression of enamel demineralization non-invasively is of high interest in conservative dentistry. By examining tooth samples with suspected occlusal lesions, we show the potential of depolarization imaging based on polarization-sensitive optical coherence tomography for the assessment of carious lesions and validate the results by co-registered X-ray micro-computed tomography volumes.
\end{abstract}

\section{Introduction}

Assessing the stage and progression of caries noninvasively and preferably without utilizing ionizing radiation is of high interest in dentistry. Besides the early detection of carious lesions, conservative therapies, aiming for the remineralization of the demineralized enamel, depend on reliable measures of the enamel state to evaluate the success of the treatment [1]. Multiple techniques for qualitative and quantitative enamel analyses in vivo have been developed, which can be applied on a regular basis as they are based on optical technologies, e.g. trans-illumination and fluorescence. However, both heterogeneity and geometry of the intraorally examined tooth structures complicate the quantitative and repeated assessment for a longterm progression analysis.

Optical coherence tomography (OCT) is a non-invasive, interferometric imaging technique based on near-infrared light, that provides depth-resolved images and volumes of semi-transparent samples. Conventional OCT images represent the backscattering properties and are thus susceptible to various characteristics and alterations of the enamel, e.g. the alignment of the enamel rods, surface stains and demineralization. Additionally exploiting the polarization changes in the sample, i.e. utilizing polarization-sensitive OCT (PS-OCT), provides a more specific measure of those characteristics. We have recently shown that depolarization imaging based on PS-OCT is able to detect early stages of enamel demineralization [2] and facilitates the differentiation of surface staining and discoloration from carious lesions [3].

To assess the validity of this contrast mechanism, we acquired data sets of tooth samples with suspected occusal lesions using PS-OCT and X-ray micro-computed

*e-mail: jonas.golde@tu-dresden.de tomography $(\mu \mathrm{CT})$, an in dental research established radiographic imaging technique for demineralization imaging. By software-based registration of the volumes, we present here the spatially aligned comparison of PS-OCTbased depolarization images and $\mu \mathrm{CT}$ sections of a human molar tooth.

\section{Methods and materials}

The $1300 \mathrm{~nm}$ swept-source PS-OCT system [2] as well as the processing [3] of the depolarization images, based on calculating the noise-immune degree of polarization, have been described previously. After PS-OCT imaging of the extracted molar tooth samples, which were collected during clinical treatment and visually inspected by an experienced dentist, $\mu \mathrm{CT}$ volumes were acquired and pre-processed using a commercial high-resolution x-ray tomograph (Bruker Skyscan 1172, $85 \mathrm{kV}$ source voltage). For all subsequent processing steps including registration and visualization, custom MATLAB implementations were used. After rescaling both volumes to uniform voxels sizes of $8 \times 8 \times 8 \mu m^{3}$, a 3D canny edge detection algorithm was used to segement the tooth surface. The surface segmentation data sets were transformed into point clouds and downsampled for speed and registration improvements. Subsequent registration of the point clouds was performed by rigid transformation based on the "iterative closest point" algorithm as implemented in the MATLAB Computer Vision Toolbox. Fig. 1 shows three representative slices of the spatially aligned volumes and a photograph of the occlusal surface. 


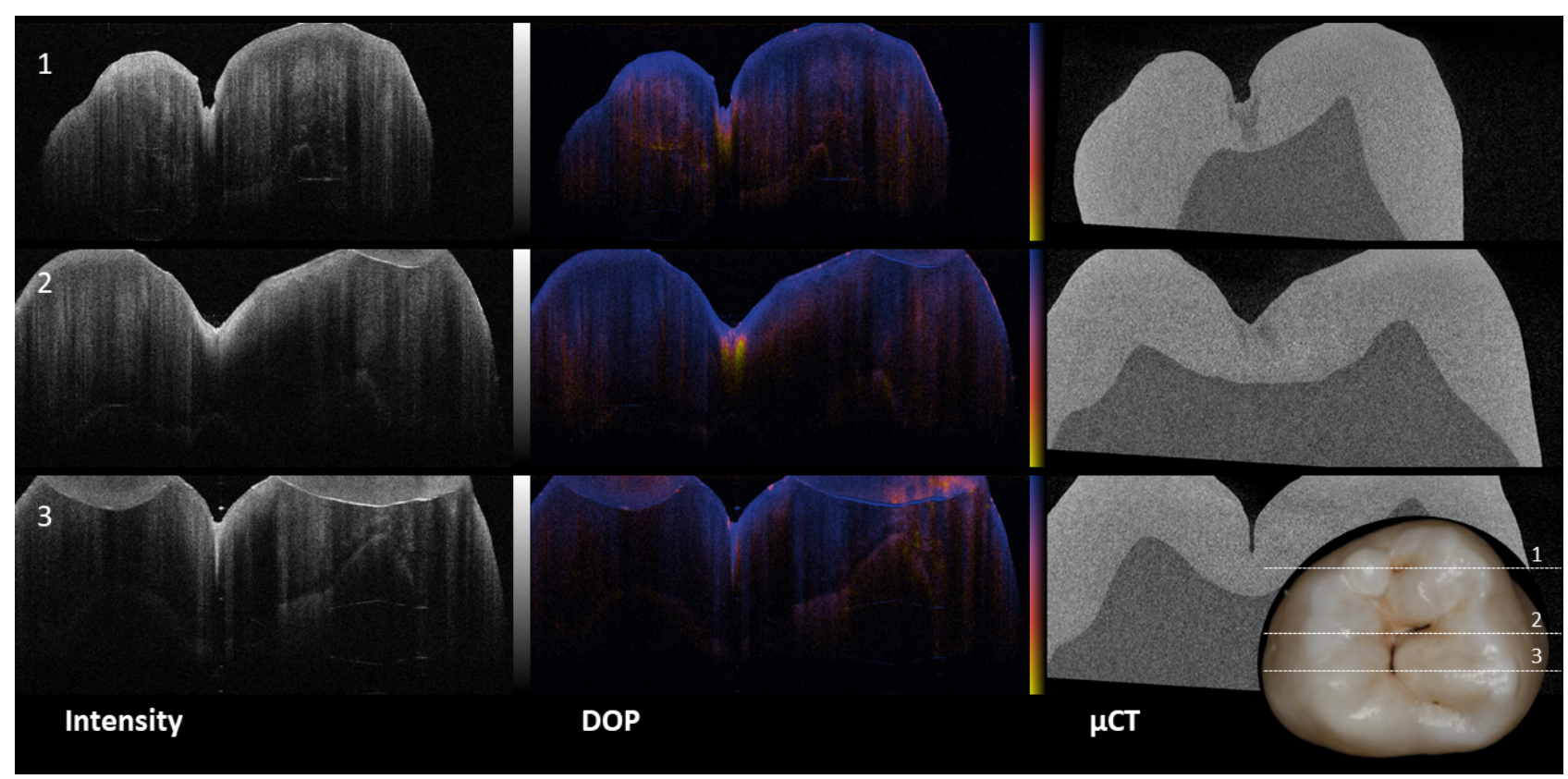

Figure 1. Comparison of PS-OCT and $\mu \mathrm{CT}$ slices from co-registered volumes: Intensity $(-2 \ldots 41 \mathrm{~dB})$, degree of polarization (DOP) with intensity overlay (DOP: $0.25 \ldots 1$, Int: $-2 \ldots 28 \mathrm{~dB}$ ) and spatially aligned $\mu \mathrm{CT}$ images, spanning $9.6 \mathrm{~mm}$ in lateral and $4.3 \mathrm{~mm}$ in axial direction. Photograph (bottom right) of the occlusal surface with slice assignments.

\section{Results and conclusion}

The presented molar tooth shows considerable stains in the occlusal fissures and indications of demineralization. Depolarization images and $\mu \mathrm{CT}$ slices of selected regions (Fig. 1) confirm the suspected demineralization in the enamel in both the outer (slice 1) and the central pit (slice 2), as reduced DOP values for PS-OCT and reduced absorption, i.e. intensity, for $\mu \mathrm{CT}$ indicate. Furthermore, both modalities are in good agreement assessing only stains but no considerable demineralization in the darkcolored fissure (slice 3). In comparison of the PS-OCT depolarization and intensity images, the more specific contrast of the DOP is highlighted as both staining and demineralization cause an altered scattering contrast in the intensity images, but DOP facilitates the differentiation.

Although the applied registration provides corresponding depth sections for both modalities, the light propagation, i.e. surface refraction and optical vs. geometric thickness of the material, was not taken into account for the alignment of $\mu \mathrm{CT}$ and PS-OCT images. Thus, the approach is currently limited to a qualitative comparison of enamel demineralization in corresponding regions. Both more sophisticated alignment approaches for the analysis of tooth samples and the examination of artificial, less complex samples with demineralization might provide the basis for the quantitative assessment of demineralization by depolarization imaging.

\section{Acknowledgments}

This project is co-financed with tax funds based on the budget decided by the members of the Saxon State Parliament. This project was supported by the European Union/ European Social Fund (ESF) and the Free State of Saxony within the ESF junior research group "Optical Technologies in Medicine" (project number 100270108). The author Jonas Golde was supported by the European Union/ European Social Fund (ESF) and the Free State of Saxony within a doctoral scholarship (project number 100284305).

\section{References}

[1] H. Meyer-Lueckel, S. Paris, Operative dentistry 41, S35 (2016)

[2] J. Golde, F. Tetschke, J. Walther, T. Rosenauer, F. Hempel, C. Hannig, E. Koch, L. Kirsten, Journal of biomedical optics 23, 071203 (2018)

[3] J. Golde, F. Tetschke, R. Vosahlo, J. Walther, C. Hannig, E. Koch, L. Kirsten, SPIE 11078, Optical Coherence Imaging Techniques and Imaging in Scattering Media III (2019), p. 24 